$\xi=-1$

\title{
TOA-based source localization using ML estimation
}

\author{
Ram Prasad Gundu ${ }^{1 *}$, P Pardhasaradhi², S Koteswara Rao ${ }^{3}$, V Gopi Tilak \\ ${ }^{1,4}$ M. Tech Student \\ ${ }^{2,3}$ Professor \\ Department of ECE, KLEF, Guntur, AP, India \\ *Corresponding author E-mail: ramprasad.gundu@gmail.com
}

\begin{abstract}
This paper proposes the Time of arrival (TOA) measurement model for finding the position of a stationary emitting source for Line-ofSight (LOS) scenario. Here Maximum Likelihood Estimation (MLE) is used as the positioning algorithm. For approximation of the roots of the solution, which directly corresponds to the source location, the optimization techniques used are Gauss-Newton, Gradient descent and Newton-Raphson methods. Two different cases are considered for investigation in this paper. The first case compares the three different optimization techniques in terms of convergence rate. In the second case the error values obtained from two different scenarios are compared, one involving a single trial only, while the second scenario uses Monte Carlo method of simulations. Firstly, the error values, for both the coordinates (two-dimensional), obtained by getting the difference between the measured source positions and the initially guessed source position are obtained for a single trial. Later using Monte Carlo simulation method, the Root-Mean-Square (RMS) error values, for both the coordinates (two-dimensional), for the optimization techniques are obtained. To improve the performance of the algorithm, Monte Carlo simulation has been used for multiple trials.
\end{abstract}

Keywords: Localization; Maximum Likelihood (ML); Monte Carlo; Optimization; Time of arrival (TOA).

\section{Introduction}

Source Localization is finding the position of an object. This step is performed in the initial stage of any positioning system. Localization gathers more prominence since identifying the position of the source, is the initial step of the positioning system, following which, other activities are performed and any errors cropping up at this initial stage would lead to large errors at later stages. There are numerous measurement models for measuring the distance between the emitting source and the receivers. The measurement models that are relevant in this context are Time of arrival (TOA), Direction of Arrival (DOA) or Angle of Arrival (AOA), Time Difference of Arrival (TDOA) and Received Signal Strength Indicator (RSSI) [1]. The line-of-sight (LOS) scenario is considered in this paper and that corresponds to the zero-mean Gaussian noise [1].

\section{TOA-based measurement model}

Considering the equation of distance between two points (x, y) and $(\mathrm{x} 1, \mathrm{y} 1)$, the radius of the circle is obtained, which in this context would be the distance between the source and the receiver. This is given by the following equation,

$$
\text { radius }=\sqrt{\left(x-x_{1}\right)^{2}+\left(y-y_{1}\right)^{2}}
$$

The total number of sensors, receivers or base nodes that are considered are four. The coordinates of the receivers are known whereas the source position is unknown, and hence an initial guess for the source position estimate is taken in terms of 2-dimensional rectangular coordinates.

The range, which is the distance between each of the receivers and the source consists of two components. One being the range component without any noise, and is denoted by the symbol d, while the other component corresponds to the noise vector, which is taken as white Gaussian noise with mean taken as zero. This corresponds to the line-of-sight. The reasonable quantity of signal-tonoise (SNR) ratio is considered. The number of iterations for the optimization techniques used, to realize the actual position of the source is specified as 40 .

The number of trials which corresponds to the number of samples in the Monte Carlo experiment are taken as 100 .

The mathematical expression for source localization of all the types of mathematical models is given by,

$$
r=g(x)+w
$$

The range component, $d$ without adding noise is given as,

$$
d_{i}=\sqrt{\left(x-x_{i}\right)^{2}+\left(y-y_{i}\right)^{2}}
$$

The range component, $r$ for finding the position that consists of both range without noise and the noise component, is given as,

$$
\begin{aligned}
& r_{T O A, i}=d_{i}+w_{T O A} \text { where } i=1,2,3, \ldots, S \\
& r_{T O A, i}=\sqrt{\left(x-x_{i}\right)^{2}+\left(y-y_{i}\right)^{2}}+w_{T O A, i} \\
& r_{T O A, i}=g_{T O A, i}(x)+w_{T O A, i}
\end{aligned}
$$


The range component for finding the position, in the matrix form is given as,

$$
r_{T O A}=\left[r_{T O A, 1}, r_{T O A, 2}, r_{T O A, 3}, \ldots, r_{T O A, S}\right]^{T}
$$

The noise component, $w$ for finding the position, in the matrix form is given as,

$$
w_{T O A}=\left[w_{T O A, 1} w_{T O A, 2} w_{T O A, 3} \ldots w_{T O A, S}\right]^{T}
$$

The distance matrix is given as,

$$
d=g_{T O A}(x)=\left[\begin{array}{l}
\sqrt{\left(x-x_{1}\right)^{2}+\left(y-y_{1}\right)^{2}} \\
\sqrt{\left(x-x_{2}\right)^{2}+\left(y-y_{2}\right)^{2}} \\
\sqrt{\left(x-x_{3}\right)^{2}+\left(y-y_{3}\right)^{2}} \\
\vdots \\
\sqrt{\left(x-x_{s}\right)^{2}+\left(y-y_{s}\right)^{2}}
\end{array}\right]
$$

The probability density function for the Time of arrival (TOA) based measurements is given as,

$$
p\left(r_{T O A}\right)=\frac{e^{\left(-\frac{1}{2}\left(r_{T O A}-d\right)^{T} \sigma_{T O A}{ }^{-1}\left(r_{T O A}-d\right)\right)}}{(2 \pi)^{S / 2} \sigma_{T O A}}
$$

\section{Maximum likelihood estimation algorithm}

The Maximum Likelihood Estimation (MLE) is used to estimate non-random parameters. Maximum Likelihood Estimation (MLE) is that estimator, for which the measurements are most likely and that which maximizes the probability of the measurement. Let $X$ be the unknown measurements which are values of a random variable with $X_{1}, X_{2}, X_{3} \ldots, X_{N}$ be its $N$ measurement values, and the sample values as $x_{1}, x_{2}, x_{3} \ldots, x_{N}$, then the likelihood function is given as,

$$
L(\theta)=\prod_{s=1}^{S} g_{Y \mid \theta}(y \mid \theta)
$$

Once the measurement values are obtained and the likelihood function is calculated, the entire parameter space needs to be searched over various values of x. Finally, the specific value has to be selected, which is maximum, and that is more probable which leads to Maximum Likelihood Estimation (MLE) expressed mathematically as follows,

$$
x=\arg \min _{x} C_{T O A}(x)
$$

The measurements distribution is known and the Maximum Likelihood Estimation (MLE) algorithm gives the maximum value of the position. The cost function denoted by $\mathrm{C}$ in case of TOA-based source localization is given as,

$$
\begin{aligned}
& C_{T O A}(x)=\left(r_{\text {TOA }}-g_{T O A}(x)\right)^{T} \sigma_{T O A}{ }^{-1}\left(r_{\text {TOA }}-g_{T O A}(x)\right) \\
& \sum_{s=1}^{S}\left(r_{T O A}-\sqrt{\left(x-x_{3}\right)^{2}+\left(y-y_{3}\right)^{2}}\right)^{2} / \sigma_{T O A, s}^{2}
\end{aligned}
$$

$J$ is the Jacobian matrix (See Appendix A). $H$ is the Hessian matrix (See Appendix A). $\nabla$ is the gradient (See Appendix A).

$$
H\left(C_{T O A}(x)\right)=\partial^{2} C_{T O A}(x) / \partial x \partial x^{T}
$$

where $C$ is the Maximum Likelihood Estimation (MLE) Cost function.

\section{Optimization techniques}

Optimization techniques can be classified in a number of ways. In the present context these techniques are divided depending on whether they require derivative evaluation. One approach that does not require derivatives are direct or non-gradient methods. The other gradient methods either ascent or descent ones fall under the other category that require derivatives. Here the gradient methods used for optimization are Gradient descent and NewtonRaphson.

\subsection{Newton-Raphson method}

Newton-Raphson method provides us with a procedure for locating the roots of a function of a variable. There are two alternate ways in which this method can be developed, one being a geometrical approach and the other one is by using Taylors series expansion. In the geometrical approach, firstly an initial guess is taken, for the value of the root, such as xi, is taken and a tangent is drawn at that point on the function which when extended to the $\mathrm{x}$-axis gives the next iteration value.

Newton-Raphson method can also be developed using Taylors series expansion. In this approach apart from locating the roots, it provided the rate of convergence of this method. Here Taylors series can also be used to estimate the error of the NewtonRaphson formula.

This method has fast local convergence rate, that is, once the neighborhood of the roots of the solution is reached, it converges to a high accuracy, and that too in very few iterations. The drawback of Newton-Raphson method is that, it involves Hessian matrix which is intensive in terms of computation, due to the presence of second derivatives, and hence is error-prone, burdensome and very expensive [3].

An alternate way of achieving this is,

$\left.x^{i+1}=x^{i}-H^{-1}\left(x^{i}\right)\right) \nabla\left(C_{T O A}\left(x^{i}\right)\right)$

The error is approximately equal to the square of the previous error.

This procedure uses an iterative approach wherein the convergence on the root is very rapid. Also, the true percent relative error during each iteration decreases much faster compared to that of other methods [4].

The demerits of this method are, firstly, convergence may not happen if the initial point is not close to the optimum one. Secondly, this method involves intense computation of the second partial derivatives of the functions as well as the matrix inversion during each iteration [2].

\subsection{Gauss-Newton method}

The Gauss-Newton is an optimization algorithm which iteratively finds the value of a variable that minimizes the sum of squares.

Starting with an initial guess, for attaining the minimum value, this algorithm uses the approach of iterations. This is mathematically expressed as below,

$$
\begin{aligned}
& x^{i+1}=x^{i}+\left(J^{T}\left(g_{T O A}\left(x^{i}\right)\right) \sigma_{T O A}{ }^{-1} J\left(g_{T O A}\left(x^{i}\right)\right)\right)^{-1} \\
& J^{T}\left(g_{T O A}\left(x^{i}\right)\right) \sigma_{T O A}{ }^{-1}\left(r_{T O A}-g_{T O A}\left(x^{i}\right)\right)
\end{aligned}
$$

where, $J$ is the Jacobian matrix. 


\subsection{Gradient descent method}

Gradient descent is a method for finding the local minimum value of a function. Taking an initial value of the root of a function, the value can be changed in many directions but for the getting the minimum value of the function, and then the gradient of the function needs to be taken. This algorithm will finally converge where the gradient is zero which is equal to the local minimum. This is mathematically expressed as follows,

$$
x^{i+1}=x^{i}-m \nabla\left(C_{T O A}\left(x^{i}\right)\right)
$$

The parameter $m$ is the step size of the function and is taken as 0.005 to ensure proper stability.

\section{Simulation results}

In this paper the simulation is performed using MATLAB software and the required graphs are plotted.

Two different cases are considered while simulating the TOAbased measurement using ML estimate. Firstly, only one trial is being considered. In this case, the error value in $\mathrm{x}$-coordinate, which is the difference between the $\mathrm{x}$-coordinate value of the initially guessed position and the $\mathrm{x}$-coordinate value of the measured position which is estimated using ML algorithm and approximated using the optimization methods is calculated and is plotted against the number of iterations. This is illustrated in Fig.1(a). In the similar fashion, the error value in y-coordinate is also calculated and is plotted against the number of iterations for single trial. This is illustrated in Fig.1(b).

Secondly, Monte Carlo method of simulation is implemented for what has been done as part of the first case. Here the calculation of the Root-Mean-Square (RMS) error values is repeated for $N$ number of trials. This is illustrated in Fig.2(a), for the x-coordinate plotted against the number of iterations and in Fig.2(b), for the ycoordinate plotted against the number of iterations. Since this case (Monte Carlo) involves large sets of data, it is stored in flat files and were later retrieved when necessary. This is performed for the sake of programming convenience.

The data for all the three optimization methods are included in the graphs for both single trial as well as the Monte Carlo method ( $N$ trials). Monte Carlo experiments in the present context gives a better performance in estimating the source position.

It has been observed that the trajectory of the estimated position of the source for Gauss-Newton and Newton-Raphson methods converges much faster than that of the Gradient descent method. This has been demonstration in the both (Fig. 1 and Fig. 2).

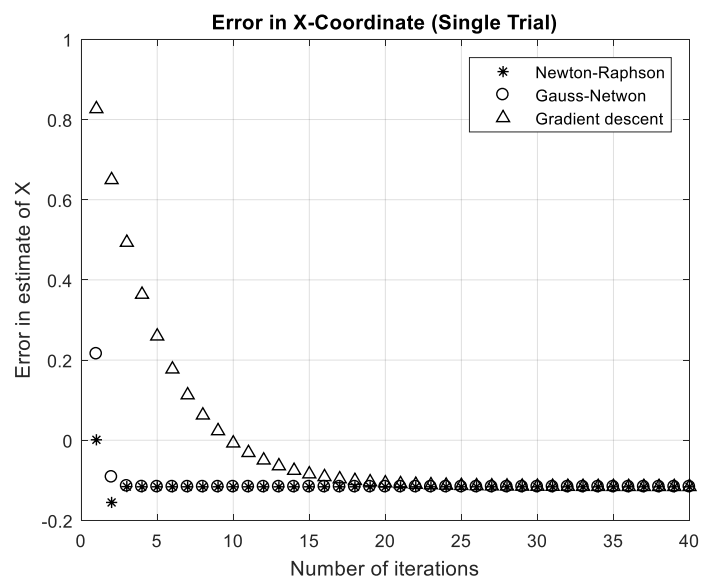

1 (a)

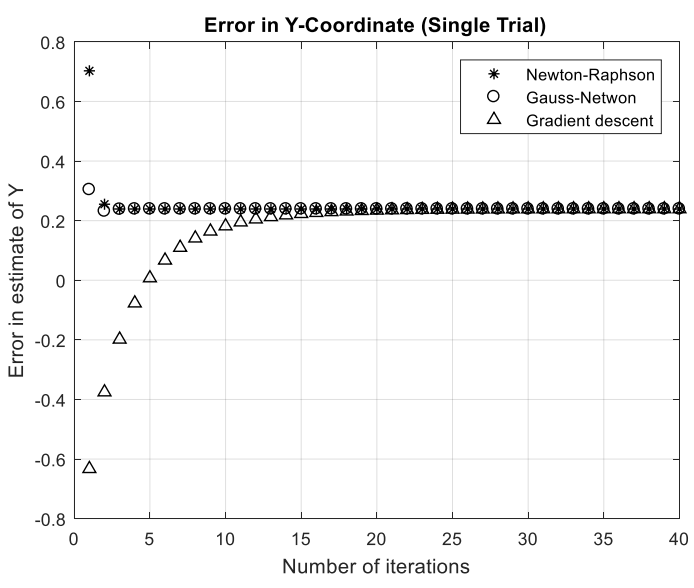

1 (b)

Fig. 1: (a) Error in X-coordinate for Single Trial (b) Error in $Y$ coordinate for Single Trial.

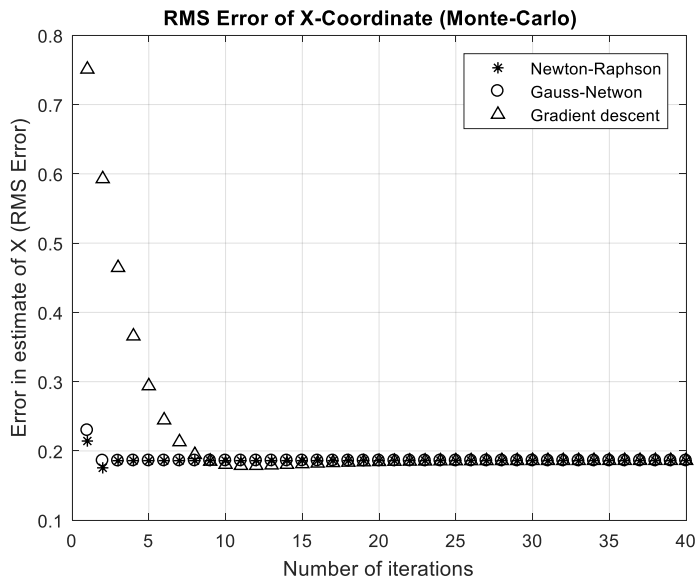

2 (a)

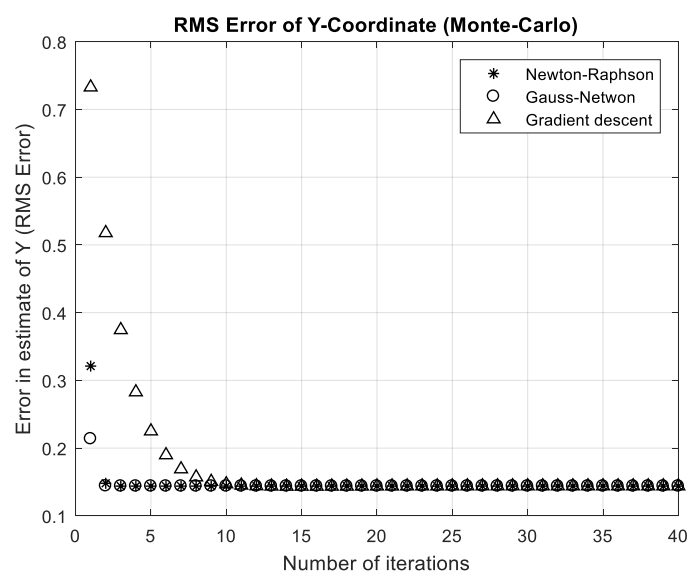

2 (b)

Fig. 2: (a) RMS error in X-coordinate for Monte Carlo method (b) RMS error in Y-coordinate for Monte Carlo method.

\section{Conclusion}

This paper proposes TOA-based mathematical model and Maximum Likelihood Estimation for determining the position of the emitting source. For approximating the real position of the source from the initial guess ML estimator is used in association with three different optimization techniques. Multiple optimization techniques are investigated in order to analyze find out the best optimization approach for source localization.

The numerical methods proposed in this paper for approximating the real position of the source are Newton-Raphson, Gradient descent, and Gauss-Newton method. In our investigation the error in the measured and the initially guessed positions are compared and hence, it has been identified that the Newton-Raphson and the 
Gauss-Newton methods converge more rapidly to the roots (corresponding to the coordinates of the position) of the equation, compare to the Gradient descent method.

This paper also proposes the Monte Carlo method of simulations for estimating the source. The relative convergence rates, of all the above-mentioned optimization methods stay the same for both the single trail and the Monte Carlo method scenarios.

It has been observed and concluded that Monte Carlo method provides better performance of the algorithm, in estimation of the source position.

There are some disadvantages of using Time of arrival (TOA) for Source Localization. Firstly, it requires both the receivers and the source to be precisely synchronized. Secondly, Time of arrival (TOA) requires the emitted signal from the source to include a time stamp, so that the receivers can determine the exact time at which the receiver has transmitted the signal. This increases the complexity of this method, with increased chances of error in finding the position of the source [1].

\section{Appendix A}

\section{A.1. Gradient}

A gradient is a vector field of a scalar function $g\left(x_{1}, x_{2}, x_{3} \ldots, x_{n}\right)$ and is mathematically represented with the symbol $\nabla g$, where the $\nabla$ represents a vector differential operator, and is pronounce as "del". A gradient is a multivariable generalization of derivative. The difference between a derivative and a gradient is that, the derivative is defined on functions of single variable, whereas the gradient is defined on functions of multiple variables. A derivative is a scalar-valued function, whereas the gradient is a vector-valued function.

The gradient of a function $g$ is given by the following expression,

$$
\operatorname{grad} g \text { or } \nabla g(x)
$$

In the 3-dimentional cartesian coordinate system the gradient is given by:

$$
\nabla g(x, y, z)=[(\partial g / \partial x) i+(\partial g / \partial x) j+(\partial g / \partial x) k]^{T}
$$

where $\mathrm{i}, \mathrm{j}$, and $\mathrm{k}$ are the unit vectors in the directions $\mathrm{x}, \mathrm{y}$ and $\mathrm{z}$ directions.

The generalized vector notation of the gradient in $\mathrm{m}$ dimensions is given as below [2]

$$
\nabla g(x)=\left[\partial g / \partial x_{1} g / x_{2} \ldots g / x_{m}\right]^{T}
$$

Gradient provides best local trajectories for multidimensional problems [2].

It also represents the directional derivative of the function, say g, and hence $\nabla g$ represents the maximum rate of change of the function $g$ [4].

\section{A.2. Jacobian}

Jacobian matrix is a matrix of first order partial derivatives of a vector-valued function $\mathrm{g}$. If the Jacobian matrix is a square matrix, then the matrix and its determinant are called the Jacobian.

The Jacobian matrix is represented by the symbol $\mathrm{J}$ and is mathematically represented by the following expression[5-6],
$J=\left(\begin{array}{ccc}\partial g_{1} / \partial x_{1} & \ldots & \partial^{2} g_{1} / \partial x_{n} \\ \vdots & \ddots & \vdots \\ \partial g_{m} / \partial x_{n} \partial x_{1} & \cdots & \partial g / \partial x_{n}\end{array}\right)$

The Jacobian matrix is the generalization of the gradient.

\section{A.3. Hessian}

The Hessian matrix is a square matrix which consists of the second partial derivatives of multivariable scalar-valued functions. This is used to describe the local curvature of a function consisting of multiple variables. It is denoted by the symbol $H$ and is mathematically represented in terms of the function $\mathrm{g}$ by the following expression.

$H=\left(\begin{array}{ccc}\partial^{2} g / \partial x_{1}^{2} & \ldots & \partial^{2} g / \partial x_{1} \partial x_{n} \\ \vdots & \ddots & \vdots \\ \partial^{2} g / \partial x_{n} \partial x_{1} & \cdots & \partial^{2} g / \partial x_{n}^{2}\end{array}\right)$

It is denoted by the symbol $\nabla^{2} g(x)^{2}$ [3]. The determinant of the Hessian matrix is denoted by the symbol $|\mathrm{H}|$ and is referred to as Hessian. One of the uses of Hessian is in optimization, in which it performs searches in order to include second order curvature to attain better results [2]

\section{References}

[1] Seyed A. (Reza) Zekavat, R. Michael Buehrer, "Handbook of Position Location - Theory, Practice, and Advances", 2012 IEEE, John Wiley \& Sons, Inc., Hoboken, New Jersey, Chapter 1,2,6,7.

[2] Steven C. Chapra, Raymond P. Canale "Numerical Methods for Engineers: with Software and Programming Applications", Tata McGraw-Hill, Fourth Edition, Chapter-6,14.

[3] Jorge Nocedal, Stephen J. Wright, "Numerical Optimization" Second Edition, Springer International Edition.

[4] B. S. Grewal Higher Engineering Mathematics, 43rd Edition, Khanna Publishers, May 2015

[5] [5] Vudatha, C.P., Nalliboena, S., Jammalamadaka, S.K.R., Duvvuri, B.K.K., Reddy, L.S.S., Automated generation of test cases from output domain of an embedded system using Genetic algorithms, ICECT 2011 - 2011 3rd International Conference on Electronics Computer Technology 5,5941989, pp. 216-220

[6] [6] Sastry, J.K.R., Ganesh, J.V., Bhanu, J.S., I2C based networking for implementing heterogeneous microcontroller based distributed embedded systems, Indian Journal of Science and Technology, Volume 8, Issue 15, 2015

[7] ANNABATTULA, J., KOTESWARA RAO, S., SAMPATH DAKSHINA MURTHY, A., SRIKANTH, K.S. and DAS, R.P., 2015. Underwater passive target tracking in constrained environment. Indian Journal of Science and Technology, 8(35), pp. $1-4$.

[8] SUNDER, P.S., KOTAMRAJU, S.K., RAMAKRISHNA, T.V., MADHAV, B.T.P., SRUTHI, T.S., VIVEK, P., KUMAR, J.J. and DILEEP, M., 2015. Novel miniatured wide band annular slot monopole antenna. Far East Journal of Electronics and Communications, 14(2), pp. 149-159.

[9] JAWAHAR, A. and KOTESWARA RAO, S., 2015. Recursive multistage estimator for bearings only passive target tracking in ESM EW systems. Indian Journal of Science and Technology, 8(26),

[10] VUNDAVILLI, P.R., PARAPPAGOUDAR, M.B., KODALI, S.P. and BENGULURI, S., 2012. Fuzzy logic-based expert system for prediction of depth of cut in abrasive water jet machining process. Knowledge-Based Systems, 27, pp. 456-464. 